\title{
An Improved MPPT Method Based on Fuzzy Logic Controller for a PV System
}

\author{
Muhannad ALSHAREEF \\ Department of Communication and Electronics Engineering, College of Engineering in Al-Qunfudhah, \\ Umm al-Qura University, Mecca, Saudi Arabia \\ mjshareef@uqu.edu.sa
}

\begin{abstract}
This paper proposes, implements and incorporates a new maximum power point tracking (MPPT) method for controlling photovoltaic systems (PV). The structure and characteristics of conventional hill-climbing based MPPT are analysed. Moreover, the simplicity of its implementation in the environment of the MATLAB software is dues to using the C-block in order to simulate the algorithm and the PV system. The proposed fuzzy logic controller (FLC) based on the variable step-size hill-climbing MPPT method provides a rapid and precise convergence to the maximum power point (MPP) under normal and varying weather conditions in comparison with the conventional hill-climbing method. The simulation results are provided in order to validate the performance and feasibility of the proposed controller, while taking into consideration different conditions for the solar irradiation operation.
\end{abstract}

Keywords: Fuzzy-logic controller (FLC), Maximum power point tracking (MPPT), Hill-climbing, Energy efficiency, Photovoltaic system.

\section{Introduction}

The growth of renewable energy sources has attracted research interest, as a result of technological developments and changes in the energy market, particularly following the depletion of fossil fuels, the energy crisis, as well as environmental issues, including pollution and global warming. Renewable energy sources such as photovoltaic solar panels, small turbines, are becoming more impetuous.

A PV system can function under various loads and system conditions in a grid-connected configuration. (Shah et al., 2020; Taşc1karaoğlu \& Erdinc, 2019). Grid-connected PV systems are widely used to inject power into a grid as part of a distributed generation system. A stand-alone PV system is preferable from a financial perspective, whereas other sources of electricity, such as those in mobile applications, transportation, and satellite networks are impossible or difficult to utilize (Hariri et al., 2020). However, PV systems have a low conversion efficiency and higher manufacturing costs relative to fossil fuel. (Ahmed \& Salam, 2016). It should be noted that the PV array's power and current characteristics are non-linear and impacted by the temperature and solar irradiance change. Hence, a maximum power point tracker (MPPT) can be used in the PV system to track the maximum power point (MPP). Several different techniques for MPPT have been proposed.

The current methods differ in terms of simplicity, precision, time response, expense and other technological aspects (Ali et al., 2020). The MPP-tracking voltage base system states that the ratio between maximum power voltage and opencircuit voltage is linearly proportional in various weather conditions (Raverkar et al., 2020). As this method relies on an estimation of a fixed value, the extracted power is probably lower than the actual MPP, causing significant power loss. In addition, this approach will not monitor MPP if such PV array cells have been partially shaded or impaired.

A current-based MPPT method was presented in (Owusu-Nyarko, Elgenedy \& Ahmed, 2019). This system calculates the ratio of maximum current to short-circuit current under various weather conditions. This method is the same as the voltage-based MPPT method. Due to its easy implementation and high monitoring precision, the incremental conductance (INC) method is usually employed. This method is based on the fact that the slope of PV power derivative over PV voltage is zero at the MPP. This approach is proposed to improve the precision and dynamic efficiency of monitoring under quickly changing conditions. However, it requires a complex calculation with the purpose of obtaining good results under different weather conditions (Ali et al., 2021; Liu et al., 2008).

A new technique known as ripple correlation control (RCC) was developed (Sahu, Sharma \& Dey, 2020). This uses the ripples of a converter signal to track the MPP. By adjusting the operating 
current as per location, the optimum point is reached. In this technique, the tracking speed is reasonably fast.

A comparison was made between MPPT methods (Ali et al., 2020; Shadlu, 2019). The perturb and observe ( $\mathrm{P} \& \mathrm{O})$ and hill-climbing $(\mathrm{HC})$ algorithms are the most popular MPPT algorithms among other MPPT methods due to their simplicity and low cost. However, these algorithms suffer from series issues, such as continually oscillating around the MPP. Furthermore, as their irradiation increases rapidly over time, the $\mathrm{P} \& \mathrm{O} / \mathrm{HC}$ is prone to losing its tracking direction (Ahmed \& Salam, 2018). In addition, there must be compromise between fast tracking and steady-state response in order to determine the suitable step-size of the perturb and observe algorithm. The trade-off problems typically need to be considered in the context of fixed stepsize MPPT methods. The situation becomes even worse in case of rapid and constant fluctuations of environmental conditions such as irradiance and temperature (Kollimalla \& Mishra, 2014) Many variable step-size MPPT methods were thus proposed to eradicate such dilemmas. However, the step-size of such procedures is dependent on panel data specifically applicable to the operating environment. Instead, advanced artificial intelligence (AI) systems are being developed by (Chiu, 2010; Alshareef et al., 2019) for extracting the maximum power in order to avoid problems related to fixed and variable step-size methods, such as neural network, fuzzy logic (FL), particle swarm optimization (PSO) or genetic algorithm.

Fuzzy logic is one of the most suitable techniques for controlling PV systems as it relies on prior knowledge of the input / output relationship of the system and does not require the use of accurate system data (Tang et al., 2017; Kamal et al., 2019). Accordingly, an important research area has led to the development and improved performance of FL-based MPPT methods. Many studies have implemented hybrid MPPT methods using FL. The best functionality of the combined methods is exhibited by these hybrid MPPT methods. A hybrid approach incorporating the FL and hillclimbing (HC) approaches has been proposed. (Bouakkaz et al., 2020). The FL control has a better performance compared to the traditional HC method. Furthermore, in (Remoaldo \& Jesus, 2021), the perturb and observe (P\&O) is presented in correlation with a new MPPT approach that is enhanced by a fuzzy logic algorithm. These methods have obtained a good trade-off between the steady-state response and the dynamic response. Nevertheless, they have proven to be more difficult to apply and have a lower reliability under rapidly changing irradiance conditions. An adaptive fuzzy logic controller has been introduced to ensure the MPP of the PV system is accurately and adaptively monitored (Rezk et al., 2019).

In this paper, a new flexible fuzzy logic controller (FLC) based on variable step-size hill-climbing MPPT method is proposed for a PV system. The weakness of the conventional hill-climbing algorithm is investigated. The proposed controller will eliminate the downsides of the hill-climbing method. The FL controller is designed according to the controller input and output and divided into five membership functions (MFs) that convert the MPPT algorithm into 25 fuzzy rules. With the purpose of testing the performance and reliability of the proposed method, simulation results are obtained. The proposed FLC based on variable step-size hill-climbing method was compared to the conventional hill-climbing controller.

The key contributions of this work can be summarized as follows:

- A new approach for flexible MPPT control is proposed, developed, and applied on the basis of the fuzzy logic control method;

- Using C-block programs in the MATLABSimulink simulation software, a simple implementation of adaptive algorithm for the fuzzy logic based on variable step-size hillclimbing MPPT method is introduced.

This paper is organized as follows: Section 2 presents the PV model and Section 3 describes the conventional hill-climbing method. Section 4 defines the proposed MPPT method, while Section 5 sets forth the use of MATLAB-Simulink software in order to verify the effectiveness of the proposed MPPT method. The conclusion of the paper is outlined in Section 6.

\section{PV Model}

PV arrays consist of PV panels, which consist of series and parallel attached PV cells. The panels are connected in series and in parallel in order to match the needed voltage, current and power. With a view to displaying the mathematical model of 
the PV array, a single diode model is employed for modelling the PV cell as it is shown in Figure 1, Equation (1), which is derived from the equivalent circuit of a cell, can be used to model the PV array where all the cells are similar (Alajmi et al., 2011).

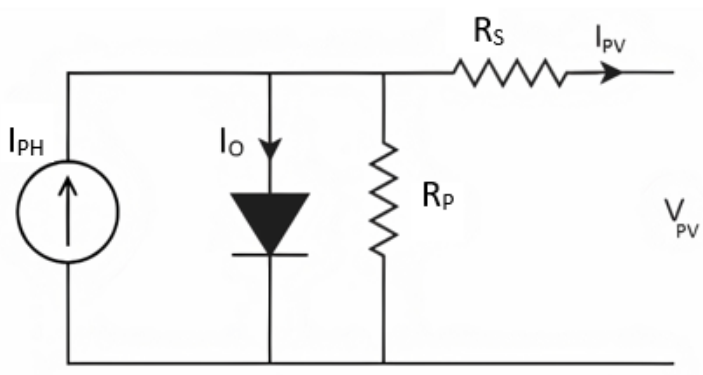

Figure 1. Equivalent circuit of PV cells (Ali et al., 2021)

$I_{P V}=N_{P} \times I_{P H}-N_{P} \times I_{O}\left[\exp \left(\frac{q\left(V_{P V}+R_{S} \times I_{P V}\right)}{A \times k \times T \times N_{S}}\right)-1\right]$

$-N_{P}\left(\frac{V_{P V}+R_{S} \times I_{P V}}{N_{S} \times R_{P}}\right)$

$V_{P V}$ and $I_{P V}$ reflect the PV array output voltage and PV array output current, respectively. $R_{S}$ and $R_{P}$ represent the series and parallel resistance of solar cells. $I_{P H}$ is the current generated by light, $I_{O}$ is the reverse current of saturation, $A$ is an ideal factor, $k$ is the Boltzmann constant, $1.38 \times 10^{-23} \mathrm{~J} \times K^{-1} . T$ is the temperature (in Kelvin degree), and $N_{S}$ and $N_{P}$ show the number of cells connected in series or in parallel, respectively. $q$ is the charge of the electron $\left(1.6 \times 10^{-19} \mathrm{C}\right)$.

Changing irradiance $G$ and temperature $T$ have a significant influence on the characteristic curves of the PV array. In addition, these changes may have significant implications for the control and design of the PV system. Figure 2 shows the characteristics of the Power-Voltage $(P-V)$ curve at different levels of irradiance and temperature.

The maximum power point can be located in a particular duty cycle, based on environmental conditions, as it can be seen in Figure 2. The $\mathrm{PV}$ array can be operated at its maximum power point by direct or indirect coupling. (Salas et al., 2006) mentioned that the PV array can be attached directly to the load using a direct coupling method and periodic fine-tuning is needed. On the other hand, the PV converter can be connected, using the indirect coupling method between the PV array and the load such that automatic monitoring of maximum power point is enabled. The MPPT algorithm is employed for controlling the power converter switch with the purpose of obtaining maximum power from the PV array. PV system structure diagram is shown in Figure 3.

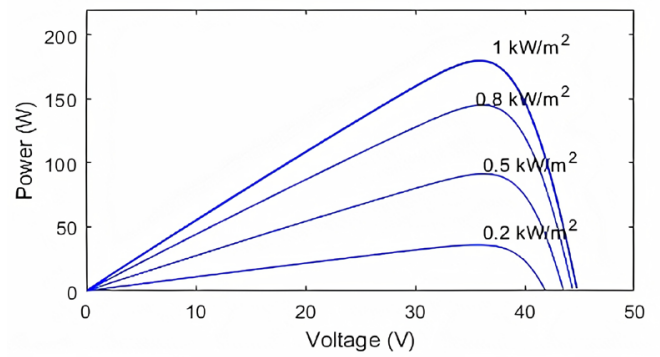

(a)

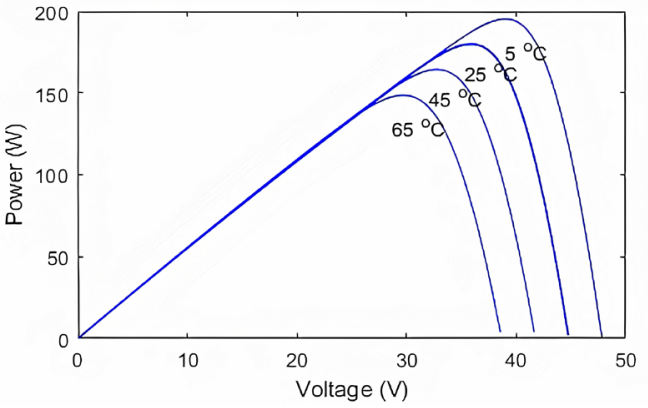

(b)

Figure 2. Influences on the characteristics of $P-V$ curve by (a) solar irradiation $(G)$ and (b) temperature $(T)$

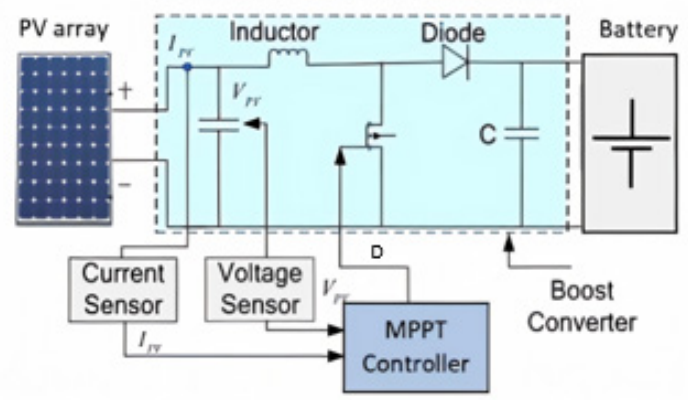

Figure 3. Schematic diagram of the PV system (Alshareef et al., 2019)

\section{Conventional Hill-Climbing Method}

The hill-climbing method perturbs the operating point of the PV system by adjusting the duty cycle of the power converter and analyses its influence on the PV output power array. The hill-climbing method has several benefits, such as a simple, fast and inexpensive implementation which make it the most common algorithm in practice. Conversely, it has three disadvantages: 1) The PV power amplitude oscillates around the MPP at normal conditions, leading to system power loses, 2) long tracking time to the MPP, 3) The operating point moves away from MPP on cloudy days when irradiance changes immediately. Figure 4 illustrates the behaviour of the PV system 
controlled through the conventional hill-climbing based MPPT method. The PV output power shown in Figure 4(a), in the context of using the hillclimbing algorithm, was forced to reach the MPP. Fluctuation around the MPP can then be noticed, after the PV power reaches the MPP. The solar irradiance is changed from $0.5 \mathrm{~kW} / \mathrm{m}^{2}$ to $1 \mathrm{~kW} / \mathrm{m}^{2}$ at $0.5 s$ thereby transferring the $\mathrm{PV}$ output power to the new MPP. Figure 4(b) displays the controller output to validate the above-mentioned drawbacks of the hill-climbing algorithm. Ellipse 1 indicates the required time to determine the converter's ideal duty cycle. The variation of the duty cycle around the ideal value is illustrated by Ellipse 2 . Ellipse 3 indicates the divergence from the ideal value of the duty cycle.

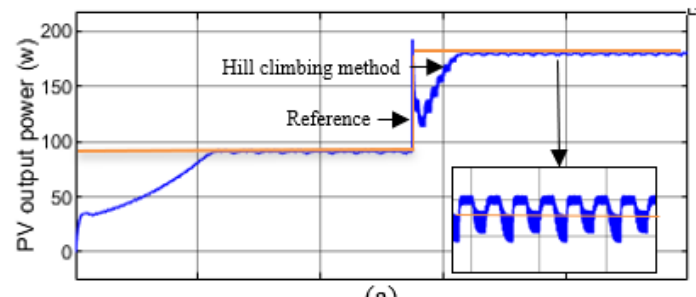

(a)

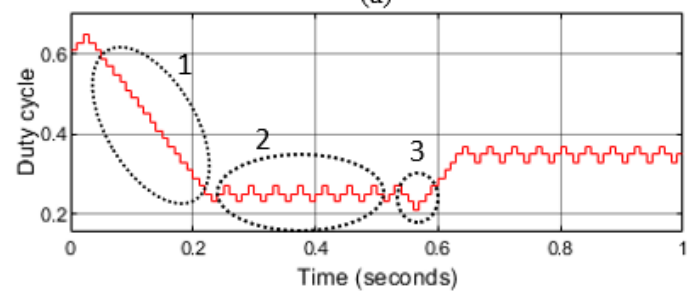

(b)

Figure 4. The hill-climbing technique's disadvantages: (a) PV output power and (b) duty cycle

Many strategies have been discussed in literature with a view to improving the hill-climbing method. A strategy to overcome the oscillation around the MPP was implemented in (Femia et al., 2005) by optimizing the time rate of sampling depending upon the converter dynamics. The second downside of the hill climbing algorithm has been substantially reduced, although the first and the third downsides remain. An adaptivebased controller was proposed to improve the steady-state and converging-speed performance (Weidong \& Dunford, 2004). The process begins with a small step in the steady state, and in the transient stage the incremental steps are increased.

To change the incremental step-size, the following equation is used:

$a(k)=M \frac{|\Delta P|}{a(k-1)}$ where $a(k)$ represents the incremental stepsize at the corresponding perturbation instant $k$, the change in power condition is expressed by $\Delta P=P(k)-P(k-1), a(k-1)$ indicates the historic value of $a(k)$, and $M$ is a constant parameter at the $k$-th perturbation.

The downside of (2) is that for the various irradiation levels and PV system, $M$ needs manual tuning, making it economically unworkable. A new $\beta$-form technique is proposed (Jain \& Agarwal, 2004; Pradeep et al., 2015). This approach uses conventional hill-climbing and the approximated $\beta$ MPP method respectively to obtain the exact MPP. The $\beta$ technique enable a quicker convergence toward the MPP than the conventional hill-climbing method. Such a system, however, relies on the parameters of the PV array, which make it impractical. An adaptive hillclimbing method is presented which is based on the application of a power window in conventional hill-climbing (Szabados \& Fangnan, 2008). The window size is tuned after each iteration loop, until the optimum power is attained. The direction of the next incremental step depends on the comparison between the recent measured power and previous measured power in the conventional MPPT method. The three-point comparison method proposed involves point A which is the current operation, point $B$ is perturbed from point $\mathrm{A}$, and point $\mathrm{C}$ is doubly perturbed from point $\mathrm{B}$ in the opposite direction (Baba, Liu \& Chen, 2020; Ying-Tung \& China-Hong, 2002). This method removes the problem of oscillation effectively; nevertheless, there is still poor convergence along with divergence problems.

\section{The Proposed MPPT Method}

This section is divided into the following two parts: part A provides a brief description of the fuzzy logic process and part B presents the proposed fuzzy logic controller (FLC) based on MPPT method.

\section{A. Brief Description of The Fuzzy Logic Controller}

The application of fuzzy logic controller is extended as it is simple, requires no mathematical modelling data and can overcome system nonlinearity. PV arrays' non-linear design and weather conditions make the tracking process very complex. Therefore FL-based MPPT methods can track MPP in the PV system with simple

https://www.sic.ici.ro 
implementation and less-needed data, as indicated in (El Khateb et al., 2014). Many FL algorithms are proposed in the previous studies to extract MPP from PV systems.

Three main stages can be used in the fuzzy logic controller's function and design. These stages are fuzzification, rule base, and defuzzification and they are shown in Figure 5. The fuzzy system has crisp input and crisp output. According to the form of membership function, the crisp input is converted into a fuzzy input through the fuzzification process. The rule base is a series of IF-THEN rules that can be derived from human or automatic creation of rules. In the fuzzy inference stage and based upon the fuzzy rules, the fuzzy output variable is provided with an implication process and an aggregate of all fuzzy outputs. The defuzzification method is then implemented to obtain the crisp output used during the control process.

\section{B. Fuzzy Logic Control Based on the Variable Step-size Hill-Climbing MPPT Method}

The main difference between the conventional fuzzy logic MPPT method and the proposed method lies in the following aspect: in the former case, the membership function limits and shapes are usually defined by the power level and power conversion system parameters, while in the latter case the membership limits and shapes are defined by a C-code that can allow easy adaptation of the membership function type.

The proposed controller is designed to take benefit of the simple implementation of hill-climbing method and eliminate all the drawbacks referred to in Section 3. The proposed controller uses hill-climbing algorithm where the step-size is controlled by the FLC-based algorithm shown in Figure 6.

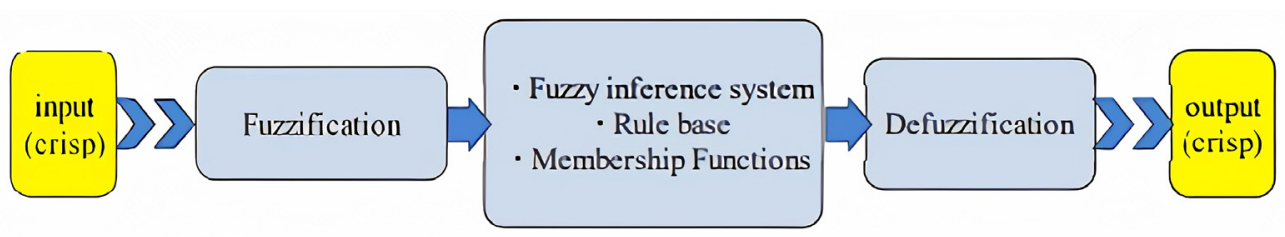

Figure 5. The basic stages of a fuzzy logic controller (Ali et al., 2021)

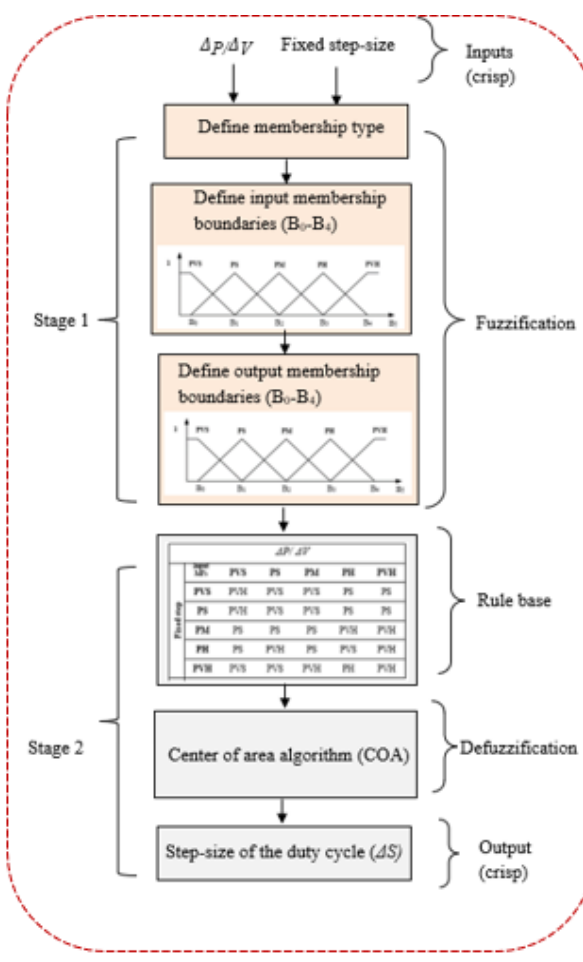

(b)

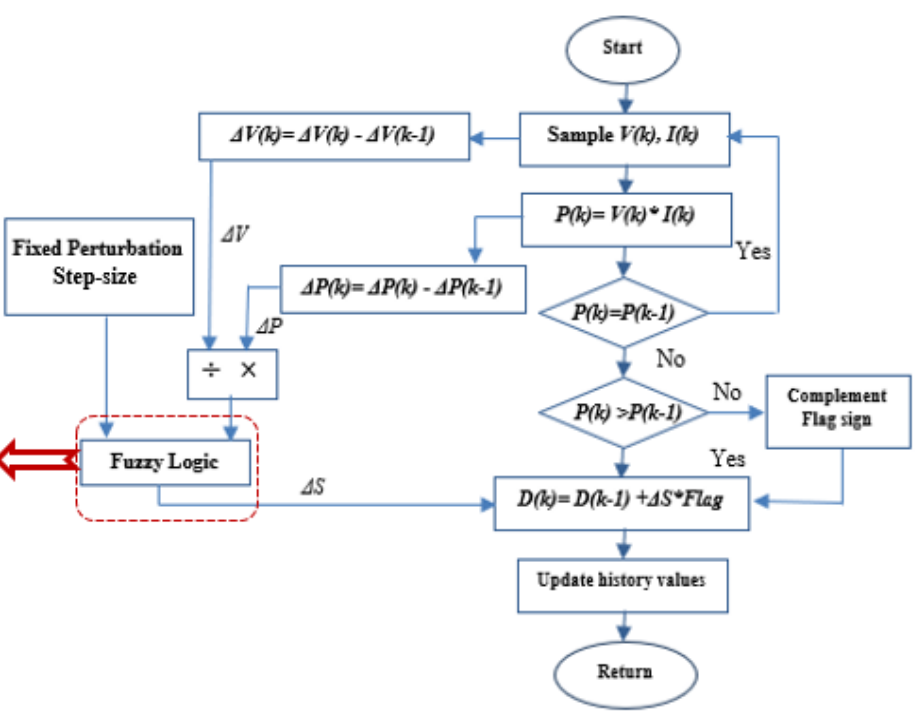

(a)

Figure 6. (a) FLC-based variable step-size hill climbing MPPT algorithm and, (b) flowchart of the proposed Fuzzy logic controller 
The inputs of FLC are: Fixed perturbation step size and the instantaneous slope $\Delta P / \Delta V$.

The following two equations can be used to determine the instantaneous slope $\Delta P / \Delta V$.

$\Delta P(i)=P(i)-P(i-1)$

$\Delta V(i)=V(i)-V(i-1)$

where $P(i)$ and $P(i-1)$ represent the current and past samples of PV measured output power. $V(i)$ and $V(i-1)$ indicate the actual and previously measured samples of the PV output voltage, accordingly.

Input and output membership functions (MFs) variables are divided into five different fuzzy subsets: $(P V S)$ positive very small, $(P S)$ positive small, $(P M)$ positive medium, $(P H)$ positive high, $(P V H)$ positive very high. In the proposed FLC controller, the implementation of the algorithm includes a total of 25 fuzzy rules. As the algorithm determines the fixed step-size and slope $\Delta P / \Delta V$ values, the FL Interface converts these values into linguistic variables (fuzzification). Then, the proposed FL controller delivers the requirement for step-size of the duty cycle to the system of output MFs. The Mamdani (Max-Min) method is employed to operate the fuzzy combination.

The final stage of the FL controller is defuzzification in which the center of area algorithm (COA) is utilized to transform changes of fuzzy subset duty cycle into actual numbers (Alajmi et al., 2011).

$$
\Delta D=\frac{\sum_{i}^{n} \mu\left(D_{i}\right) D_{i}}{\sum_{i}^{n} \mu\left(D_{i}\right)}
$$

where the FL controller output is represented by $\Delta D$ and $D_{i}$ indicates the Max-Min composition center of the output membership function.

Table 1 indicates rules of the proposed FL controller for the input and output MPs.

In Figure 6(a), the inputs of fuzzy logic controller block are the fixed step-size perturbation and the slope $\Delta P / \Delta V$, and the control changes the hill- climbing step-size $(\Delta S)$ according to the irradiance and temperature conditions.

The current power $P(k)$ is compared with prior power $P(k-1)$ in order to compute the changes in output power. According to the result of the comparison, if the change in power is positive, the perturbation of the duty cycle $D$ will move in the same direction. If not, the perturbation of the duty cycle $D$ will move in the opposite direction. The operating point would progressively shift towards the MPP by repeating the procedure.

It is important to mention that, the step-size $(\Delta S)$ is not fixed and is determined by the proposed fuzzy logic controller block. The idea of the proposed fuzzy logic control is to change the stepsize value to the location of the operating point. When the operating point is far from the MPP, the fuzzy logic controller block adjusts the step-size to a large value; if the operating point is closer to the MPP, step-size is changed to a small value. This process continues until the MPP is reached, and the proposed fuzzy logic controller set the stepsize to nearly zero. This ensures a quick dynamic response and prevents oscillations around the MPP when a steady state is attained.

Figure 6(b) shows the flowchart of the proposed FL controller (the membership function limits and shapes are defined through $\mathrm{C}$-code). The two inputs, that is instantaneous slope $\Delta P / \Delta V$ and fixed step-size can be used to define the parameters of the proposed FLC controller. In Stage 1 the membership type and limits are defined according to the required system response (fuzzification). In Stage 2, the estimated parameters and the specified membership functions are incorporated into the proposed FL controller, which outputs the duty cycle for the power converter.

This kind of fuzzy structure is used by (Rezk et al., 2019). However, this paper used this structure with a different MPPT algorithm.

Figure 7 displays the triangular membership functions for input and output MPs after the simulation of the PV system and after the input and output performance of the controller has been tested.

https://www.sic.ici.ro 


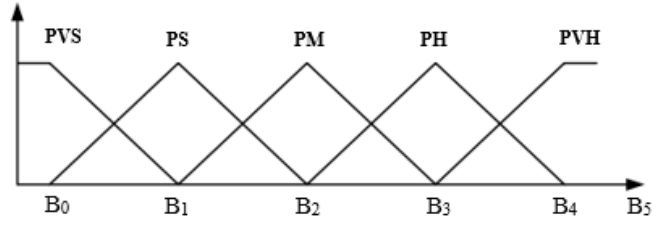

Figure 7. Membership function for input and output MPs

The $B_{0}, B_{1}, \ldots, B_{5}$ points in Figure 7 can be statically or dynamically modified in conjunction with the needed response. This allows the MPPT controller to be adapted to its implementation.

Table 1. The rules of the FL for the input and output MPs

\begin{tabular}{|c|c|c|c|c|c|c|}
\hline & & & & $\Delta V$ & & \\
\hline & $\begin{array}{l}\text { Input } \\
\text { MFs }\end{array}$ & PVS & PS & PM & PH & PVH \\
\hline . ํㅐ & PVS & PVH & PVS & PVS & PS & PS \\
\hline 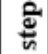 & PS & PVH & PVS & PVS & PS & PS \\
\hline . & PM & PS & PS & PS & PVH & PVH \\
\hline & PH & PS & PVH & PS & PVS & PVH \\
\hline & PVH & PVS & PVS & PVH & $\mathrm{PH}$ & PVH \\
\hline
\end{tabular}

\section{Results and Discussion}

In this section, the simulation program is employed with the purpose of validating the performance of proposed MPPT method. The program MATLABSimulink is used for modelling and testing of performance of the proposed FLC based on variable step-size hill-climbing method. The S-Function block is employed in order to insert the proposed controller algorithm in the form of C-code to allow fast adaptation of the membership functions type. The software allows measurable comparison of the efficiency and the convergence time between the hill-climbing method and the proposed method; and the PV system illustrated previously in Figure 3 is simulated. The PV voltage and the current are used as input for the MPPT controller to monitor the MPP. The output of the MPPT controller is the gate pulse used for operating a DC-DC converter's IGBT switch. The PV system specification used in the MATLAB simulation is defined in Table 2 .

Firstly, the proposed method is tested under standard conditions at an irradiation level of 1000 $\mathrm{W} / \mathrm{m}^{2}$. Figure 8 illustrates the PV output power for the hill-climbing method and for the proposed method. The tracking time for the proposed FLC method was less than a 0.01 second $(s)$. The proposed method moves the operating point more quickly toward MPP than the hillclimbing method, which means that the proposed method features a quicker convergence time. In comparison with the proposed FLC method for tracking MPP, the hill-climbing MPPT method took around $0.04 s$. In addition, there are large continuous variations in the waveform of PV output power. It is worth mentioning that the PV output power oscillations produced by the proposed method are significantly lower than the long-term oscillations observed in case of the hill climbing method.

Table 2. Specification of PV system used in MATLAB simulation

\begin{tabular}{|c|c|}
\hline Item & Value \\
\hline Maximum Power $\left(\mathrm{P}_{\max }\right)$ & $180 \mathrm{~W}$ \\
\hline $\begin{array}{c}\text { Open Circuit Voltage }\left(\mathrm{V}_{\mathrm{cc}}\right) \\
\text { Maximum Power Voltage } \\
\left(\mathrm{V}_{\text {mmpp }}\right)\end{array}$ & $44.8 \mathrm{~V}$ \\
\hline $\begin{array}{c}\text { Short Circuit Current }\left(\mathrm{I}_{\mathrm{sc}}\right) \\
\text { Maximum Power Current }\left(\mathrm{I}_{\mathrm{mpp}}\right)\end{array}$ & $5.86 \mathrm{~V}$ \\
\hline Battery & $48 \mathrm{~V}, 33 \mathrm{Ah}$ \\
\hline Switching Frequency & $10 \mathrm{KHz}$ \\
\hline Inductor & $400 \mathrm{uH}$ \\
\hline Capacitor & $1 \mathrm{~m} \mathrm{~F}$ \\
\hline Battery & $48 \mathrm{~V}, 33 \mathrm{Ah}$ \\
\hline
\end{tabular}

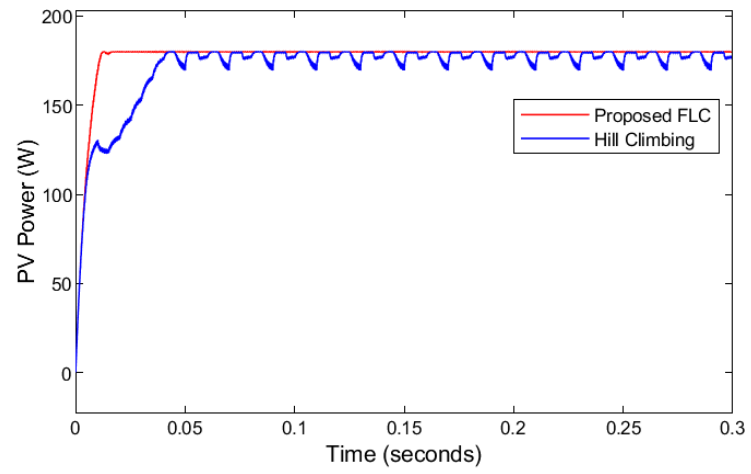

Figure 8. Simulation result of the proposed FLC based on variable step-size hill-climbing MPPT method and hill-climbing method under $1000 \mathrm{~W} / \mathrm{m}^{2}$

These two methods are compared under fast irradiation conditions, changing from 1000 $W / m^{2}$ to $800 \mathrm{~W} / \mathrm{m}^{2}$ to $600 \mathrm{~W} / \mathrm{m}^{2}$ and then $400 \mathrm{~W} / \mathrm{m}^{2}$ at the sample rate of $0.25 \mathrm{~s}$, as it is shown in Figure 9. The hill-climbing MPPT method provides an insufficient result over a large oscillation around MPP, with a longer convergence time. These 
are two of the main drawbacks of the hill-climbing method, as it was explained earlier in Section 3.

The two disadvantages are inherently eliminated by the FLC, since the decremental and incremental step-size vary in accordance with the fuzzy rules, hence the step-size is increased when the operating point is away from the MPP and vice versa.

Under gradually changing irradiation conditions, the proposed method is tested. Solar irradiance is steadily increasing/decreasing and requires a properly designed MPPT controller. In the context of a linear increase in irradiation, from 500 $W / m^{2}$ to $550 \mathrm{~W} / \mathrm{m}^{2}$ at $0.5 \mathrm{~s}$, the proposed FLC method was then evaluated. The result shown in Figure 10 demonstrated that the proposed method can follow the slow-changing irradiances acutely, allowing the PV system to attain maximum power during its entire operation.

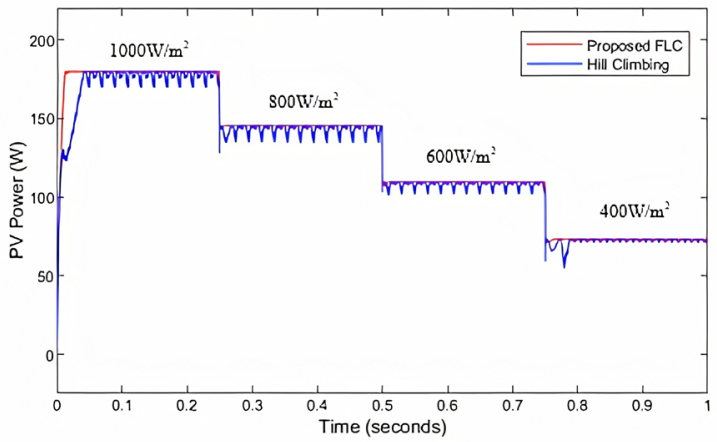

Figure 9. Simulation result of the proposed FLC based on variable step-size hill-climbing MPPT method and hill-climbing method under fast irradiation conditions

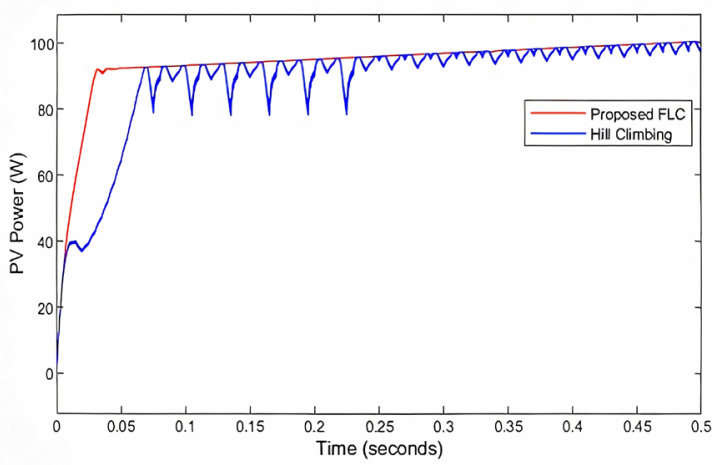

Figure 10. Simulation result of the proposed FLC based on variable step-size hill-climbing MPPT method and hill-climbing method under gradual irradiation conditions

Clearly, it is easy for the proposed FLC to track irradiance change over a limited period. The proposed controller could also mitigate the variability of the hill-climbing method. Therefore, the findings of the simulations indicated that the proposed method accomplished fast tracking with low variations around MPP during the steady-state condition. Additionally, the proposed method also offers a good trade-off between the steady-state response and the dynamic response.

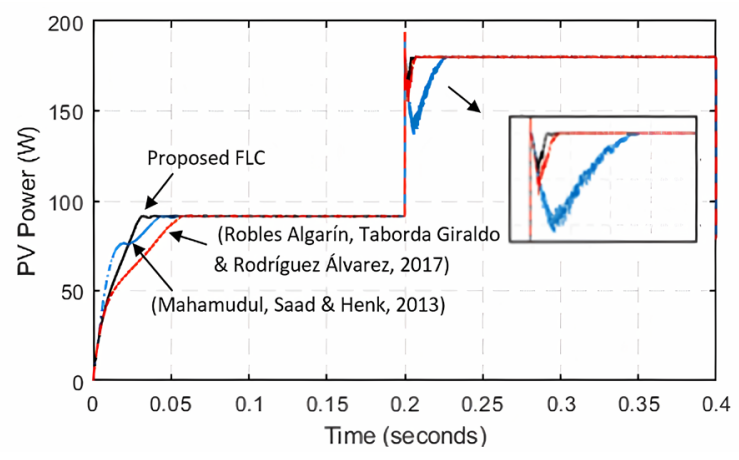

Figure 11. Simulation result of the proposed FLC based on variable step-size hill-climbing MPPT method and two others MPPT methods

A comparison has been made in order to illustrate the effectiveness of the proposed FLC method in Figure 11. The proposed FLC based on variable step-size hill-climbing MPPT method is presented in comparison with two others previously developed FLCs. The high tracking speed and the low fluctuation around the MPP are offered by both technologies. Nevertheless, in (Mahamudul, Saad \& Henk, 2013), the FLC does not track the new MPP directly, and diverges from MPP under various weather conditions. This may lead to a failure in tracking the MPP during rapidly changing weather conditions. In addition, FLC operates by using the change of PV power derivative over the PV current derivative as inputs. This could lead to a difficult operation of the controller, particularly in a noisy environment. Table 3 illustrates the additional validation of the considered method by comparing the proposed FLC based on variable step-size hill-climbing MPPT method with the existing MPPT methods.

Table 3. Compared performance of the MPPT methods

\begin{tabular}{|c|c|c|c|c|}
\hline Method & \begin{tabular}{|c|} 
Dynamic \\
performance
\end{tabular} & \begin{tabular}{|l} 
Tracking \\
accuracy
\end{tabular} & $\begin{array}{c}\text { Tracking } \\
\text { speed }\end{array}$ & Flexibility \\
\hline $\begin{array}{l}\text { (Kottas, Boutalis } \\
\text { \& Karlis, 2006). }\end{array}$ & Good & Good & High & No \\
\hline $\begin{array}{c}\text { (Mahamudul, Saad } \\
\text { \& Henk, 2013) }\end{array}$ & Bad & Good & Medium & No \\
\hline (Tang et al., 2017) & Good & Good & Low & No \\
\hline $\begin{array}{l}\text { (Robles Algarín, } \\
\text { Taborda Giraldo \& } \\
\text { Rodriguez } \\
\text { Alvarez, 2017) }\end{array}$ & Good & Bad & Low & No \\
\hline (Larbes, 2009) & Good & Good & Medium & No \\
\hline Proposed & Good & Good & High & yes \\
\hline
\end{tabular}


As it is illustrated in Table 3, the proposed method provides a fast tracking speed, great accuracy and no oscillation around MPP.

\section{Conclusion}

This paper proposes a new FLC based on variable step-size hill-climbing MPPT method for PV systems. The main benefits of the proposed method are the accurate and adaptive tracking performance of the MPP and the elimination of power variations around the MPP in transient and steady-state conditions. In addition, the proposed FLC, based on variable step-size hillclimbing MPPT method, performs a faster MPPT convergence and can be easily implemented. The proposed method could easily overcome the drawbacks of existing MPPT techniques in the literature. In the MATLAB-Simulink environment, the proposed method was applied via a C-block. The SIMULINK model was employed for verifying the findings of both the conventional hill-climbing method and the proposed FLC based on variable step-size hill-climbing MPPT method. The results of the proposed method indicate faster convergence and a lower oscillation around the MPP, and there is no deviation from the MPP under various weather conditions.

\section{Acknowledgments}

The author, Muhannad Jameel Alshareef, acknowledges the funding support received from Umm Al-Qura University, Mecca, Saudi Arabia.

\section{REFERENCES}

Ahmed, J. \& Salam, Z. (2016). A Modified P\&O Maximum Power Point Tracking Method with Reduced Steady-State Oscillation and Improved Tracking Efficiency, IEEE Transactions on Sustainable Energy, 7(4), 1506-1515.

Ahmed, J. \& Salam, Z. (2018). An Enhanced Adaptive P\&O MPPT for Fast and Efficient Tracking Under Varying Environmental Conditions, IEEE Transactions on Sustainable Energy, 9(3), 1487-1496.

Alajmi, B., Ahmed, K., Finney, S. \& Williams, B. (2011). Fuzzy-Logic-Control Approach of a Modified Hill-Climbing Method for Maximum Power Point in Microgrid Standalone Photovoltaic System, IEEE Transactions on Power Electronics, 26(4), 1022-1030.

Ali, A., Almutairi, K., Padmanaban, S., Tirth, V., Algarni, S., Irshad, K., Islam, S., Zahir, M., Shafiullah, M. \& Malik, M. (2020). Investigation of MPPT Techniques Under Uniform and Non-Uniform Solar Irradiation Condition-A Retrospection, IEEE Access, $8,127368-127392$.

Ali, M., Mahmoud, K., Lehtonen, M. \& Darwish, M. (2021). An Efficient Fuzzy-Logic Based Variable-Step Incremental Conductance MPPT Method for Gridconnected PV Systems, IEEE Access, 9, 26420-26430.

Alshareef, M., Lin, Z., Ma, M. \& Cao, W. (2019). Accelerated Particle Swarm Optimization for Photovoltaic Maximum Power Point Tracking under Partial Shading Conditions, Energies, 12(4), 623.

Baba, A., Liu, G. and Chen, X. (2020). Classification and Evaluation Review of Maximum Power Point Tracking Methods, Sustainable Futures, 2, 100020.
Bouakkaz, M., Boukadoum, A., Boudebbouz, O., Attoui, I., Boutasseta, N. \& Bouraiou, A. (2020). Fuzzy Logic based Adaptive Step Hill Climbing MPPT Algorithm for PV Energy Generation Systems. InPproceedings of the 2020 International Conference on Computing and Information Technology (ICCIT-1441), Tabuk, Saudi Arabia, (pp. 1-5).

Chiu, C. (2010). T-S Fuzzy Maximum Power Point Tracking Control of Solar Power Generation Systems, IEEE Transactions on Energy Conversion, 25(4), 1123-1132.

El Khateb, A., Rahim, N., Selvaraj, J. \& Uddin, M. (2014). Fuzzy-logic controller-based SEPIC converter for maximum power point tracking, IEEE Transactions on Industrial Electronics, 50(4), 2349-2358.

Femia, N., Petrone, G., Spagunolo, G. \& Vitrlli, M. (2005). Optimization of perturb and observe maximum power point tracking method, IEEE Transactions on Power Electronics, 20(4), 963-973.

Jain, S. \& Agarwal, V. (2004). A new algorithm for rapid tracking of approximate maximum power point in photovoltaic systems, IEEE Power Electronics Letters, 2(1), 16-19.

Hariri, M., Mat Desa, M., Masri, S. \& Mohd Zainuri, M. (2020). Grid-Connected PV Generation System - Components and Challenges: A Review, Energies, 13(17), 4279.

Kamal, T., Karabacak, M., Hassan, S., Li, H. \& Fernandez-Ramirez, L. (2019). A Robust Online Adaptive B-Spline MPPT Control of Three-Phase Grid-Coupled Photovoltaic Systems Under Real Partial Shading Condition, IEEE Transactions on Energy Conversion, 34(1), 202-210. 
Kottas, T. L., Boutalis, Y. S. \& Karlis, A. D. (2006). New maximum power point tracker for PV arrays using fuzzy controller in close cooperation with fuzzy cognitive networks, IEEE Transactions on Energy Conversion, 21(3), 793-803.

Kollimalla, S. \& Mishra, M. (2014). Variable Perturbation Size Adaptive P\&O MPPT Algorithm for Sudden Changes in Irradiance, IEEE Transactions on Sustainable Energy, 5(3), 718-728.

Larbes, C., Ait Cheikh, S. M., Obeidi, T. \& Zerguerras, A. (2009). Genetic algorithms optimized fuzzy logic control for the maximum power point tracking in photovoltaic system, Renewable Energy, 34(10), 2093-2100.

Liu, F., Duan, S., Liu, F., Liu, B. \& Kang, Y. (2008). A Variable Step Size INC MPPT Method for PV Systems, IEEE Transactions on Industrial Electronics, 55(7), 2622-2628.

Mahamudul, H., Saad, M. \& Henk, M. I. (2013). Photovoltaic System Modelling with Fuzzy Logic Based Maximum Power Point Tracking Algorithm, International Journal of Photoenergy, 762946.

Owusu-Nyarko, I., Elgenedy, M. A. \& Ahmed, K. (2019). Combined Temperature and Irradiation Effects on the Open Circuit Voltage and Short Circuit Current Constants for Enhancing their Related PVMPPT Algorithms. In IEEE Conference on Power Electronics and Renewable Energy (CPERE), Aswan, Egypt, (pp. 343-348).

Pradeep, K.P.J., Reddy, K. S. P., Mouli, C. C. \& Raju, K. N. (2015). Design and Implementation of Maximum Power Point Tracking in Photovoltaic Systems, International Journal of Engineering and Science Invention, 4(3), 37-43.

Raverkar, U. Chauhan, V. Singh \& Rani, A. (2020). An Improved Fractional Voltage Based MPPT Technique for PV Generation System. In Proceedings of the 2020 International Conference on Advances in Computing, Communication \& Materials (ICACCM), Dehradun, India (pp. 267-271).

Remoaldo, D. \& Jesus, I. (2021). Analysis of a Traditional and a Fuzzy Logic Enhanced Perturb and Observe Algorithm for the MPPT of a Photovoltaic System, Algorithms, 14(1), p. 24.

Rezk, H., Aly, M., Al-Dhaifallah, M. \& Shoyama, M. (2019). Design and Hardware Implementation of New Adaptive Fuzzy Logic-Based MPPT Control Method for Photovoltaic Applications, IEEE Access, 7, 106427-106438.
Robles Algarín, C., Taborda Giraldo J. \& Rodríguez Álvarez, O. (2017). Fuzzy Logic Based MPPT Controller for a PV System, Energies, 10(12), 2036.

Sahu, P., Sharma, A. \& Dey, R. (2020). Ripple Correlation Control Maximum Power Point Tracking for Battery Operated PV Systems: A Comparative analysis. In Proceedings of the 2020 IEEE International IOT, Electronics and Mechatronics Conference (IEMTRONICS), Vancouver, BC, Canada (pp. 1-6)

Salas, V., Ulias, E., Barrado, A. \& Lazaro, A. Review of the maximum power point tracking algorithms for stand-alone photovoltaic systems, Solar Energy Materials and Solar Cells, 90(11), 1555-1578.

Shadlu, M. (2019). Comparison of Maximum Power Point Tracking (MPPT) Algorithms to Control DCDC Converters in Photovoltaic Systems. Recent Advances in Electrical \& Electronic Engineering (formerly Recent Patents on Electrical \& Electronic Engineering), 12(4), 355-367.

Shah, M. L., Dhaneria, A., Modi, P.S., Khambhadiya, H. \& D, K. K. (2020). Fuzzy Logic MPPT for Grid Tie Solar Inverter. In tProceedings of the 2020 IEEE International Conference for Innovation in Technology (INOCON), Bengaluru, India (pp. 1-6).

Szabados, B. \& Fangnan, W. (2008). A maximum power point tracker control of a photovoltaic system. In Proceedings of the IEEE International Instrumentation and Measurement Technology Conference (pp.1074-1079).

Tang, S., Sun, Y., Chen, Y., Zhao, Y., Yang, Y. \& Szeto, W. (2017). An Enhanced MPPT Method Combining Fractional-Order and Fuzzy Logic Control, IEEE Journal of Photovoltaics, 7(2), 640-650.

Taşcıkaraoğlu, A. \& Erdinc, O. (2019), Pathways to a smarter power system. Academic press.

Weidong, X. \& Dunford, W. G. (2004). A modified adaptive hill climbing MPPT method for photovoltaic power systems. In Proceedings of the 35th Annual Power Electronics Specialist Conference (pp.1957-1963).

Ying-Tung, H. \& China-Hong C. (2002). Maximum power tracking for photovoltaic power system. In Proceedings of the 37th Industry Applications Society Annual Meeting (IAS), (pp. 1035-1040). 